OPEN ACCESS

Edited by:

Erik Rytting,

University of Texas

Medical Branch, USA

Reviewed by:

Matthew McLaughlin,

Children's Mercy Hospital, USA

PeiFan (Jane) Bai,

US Food and Drug Administration,

USA

${ }^{*}$ Correspondence:

Irina Burd

iburd@jhmi.edu

tThese authors have contributed equally to this work.

Specialty section:

This article was submitted to Obstetric and Pediatric

Pharmacology,

a section of the journal

Frontiers in Pediatrics

Received: 06 December 2016

Accepted: 28 April 2017

Published: 22 May 2017

Citation:

Lei J, Paules C, Nigrini E, Rosenzweig JM, Bahabry R, Farzin A, Yang S, Northington FJ, Oros $D$,

McKenney S, Johnston MV, Graham EM and Burd I (2017) Umbilical Cord Blood NOS1 as a

Potential Biomarker of Neonatal Encephalopathy.

Front. Pediatr. 5:112.

doi: 10.3389/fped.2017.00112

\section{Umbilical Cord Blood NOS1 as a Potential Biomarker of Neonatal Encephalopathy}

\author{
Jun Lei ${ }^{1+}$, Cristina Paules ${ }^{2 \dagger}$, Elisabeth Nigrini ${ }^{1}$, Jason M. Rosenzweig ${ }^{1}$, Rudhab Bahabry ${ }^{1}$, \\ Azadeh Farzin ${ }^{3}$, Samuel Yang ${ }^{4}$, Frances J. Northington ${ }^{1,3,5}$, Daniel Oros ${ }^{2}$, \\ Stephanie McKenney ${ }^{1}$, Michael V. Johnston ${ }^{5,6,7}$, Ernest M. Graham ${ }^{1,5}$ and Irina Burd ${ }^{1,5,6,7 *}$ \\ 'Integrated Research Center for Fetal Medicine, Department of Gynecology and Obstetrics, Johns Hopkins University \\ School of Medicine, Baltimore, MD, USA, '2Aragón Health Research Institute, SAMID Network ref RD12/0026/001, \\ Zaragoza, Spain, ${ }^{3}$ Department of Pediatrics, Johns Hopkins University School of Medicine, Baltimore, MD, USA, \\ ${ }^{4}$ Department of Emergency Medicine, Johns Hopkins University School of Medicine, Baltimore, MD, USA, ${ }^{5}$ Neurosciences \\ Intensive Care Nursery Program, Johns Hopkins University School of Medicine, Baltimore, MD, USA, ${ }^{6}$ Department of \\ Neurology, Johns Hopkins University School of Medicine, Baltimore, MD, USA, ${ }^{7}$ Department of Neurosciences, Kennedy \\ Krieger Institute, Johns Hopkins University School of Medicine, Baltimore, MD, USA
}

Background: There are no definitive markers to aid in diagnosis of neonatal encephalopathy (NE). The purpose of our study was (1) to identify and evaluate the utility of neuronal nitric oxide synthase (NOS1) in umbilical cord blood as a NE biomarker and (2) to identify the source of NOS1 in umbilical cord blood.

Methods: This was a nested case-control study of neonates $>35$ weeks of gestation. ELISA for NOS1 in umbilical cord blood was performed. Sources of NOS1 in umbilical cord were investigated by immunohistochemistry, western blot, ELISA, and quantitative PCR. Furthermore, umbilical cords of full-term neonates were subjected to $1 \%$ hypoxia ex vivo.

Results: NOS1 was present in umbilical cord blood and increased in NE cases compared with controls. NOS1 was expressed in endothelial cells of the umbilical cord vein, but not in artery or blood cells. In ex vivo experiments, hypoxia was associated with increased levels of NOS1 in venous endothelial cells of the umbilical cord as well as in ex vivo culture medium.

Conclusion: This is the first study to investigate an early marker of NE. NOS1 is elevated with hypoxia, and further studies are needed to investigate it as a valuable tool for early diagnosis of neonatal brain injury.

Keywords: neonatal encephalopathy, biomarkers, umbilical veins, NOS1, diagnosis

\section{INTRODUCTION}

Neonatal encephalopathy (NE) affects 1-9 out of every 1,000 newborns $(1,2)$ and has a fatality rate of $9-70 \%(1,3,4)$; yet, it is still poorly diagnosed at birth. Clinically, NE may manifest as a subnormal level of consciousness or seizures, and is often accompanied by difficulty with initiating and maintaining respiration and depression of tone and reflexes (5). The extent of the brain injury 
is later assessed by cranial ultrasound and MRI (6). Children diagnosed by clinical and neuroimaging criteria are at an increased risk of developing adverse long-term neurologic sequelae such as seizures and cognitive and motor difficulties (7).

Therapeutic hypothermia is the major medical treatment with promising long-term neurodevelopmental results, and this treatment is effective only when it is administered in the first six postnatal hours (8). Even with this treatment, approximately $40 \%$ of patients are affected with sequelae (8). A significant challenge in a timely treatment of NE is that the diagnosis and classification of the severity of the condition are often made only after the brain injury has already occurred. Based on easily accessible material, such as umbilical cord blood, early and prompt diagnosis of NE and associated prognosis would allow early intervention with hypothermia (9), erythropoietin (10), collection of umbilical cord blood for autologous stem cell transplantation (11), or stratification to other emerging therapies. Furthermore, provision of information to the injured neonates' families, who may face difficult therapeutic decisions, is a key for early intervention.

Given that $\mathrm{NE}$ is a rare diagnosis, studies implementing ancillary markers for the diagnosis of the condition are limited. Several biomarkers such as glial fibrillary acidic protein (GFAP), S100 calcium-binding protein B (S100B), neuron-specific enolase (NSE), adrenomedullin, and activin A were investigated, but none of them presented an opportunity for early diagnosis (12-15). A plausible candidate early marker of neurologic injury in neonates is neuronal nitric oxide synthase (NOS1). The association of the enzyme with adverse neurodevelopment is supported by animal studies $(16,17)$. In addition to neurons, NOS1 expression has been observed in non-neuronal tissues such as skeletal muscle, smooth muscle, and endothelial cells to control muscle contractility and local blood flow (18-20). Furthermore, studies have shown that NOS1 is expressed in cardiomyocytes in response to oxidative stress (21).

We hypothesized that NOS1 in umbilical cord blood is a plausible clinical biomarker for identification of NE due to hypoxic-ischemic events. To test our hypothesis, we pursued four aims: (1) to determine whether NOS1 is present not only in the umbilical cord but also in the umbilical cord blood of neonates; (2) to determine whether the level of NOS1 in neonates with a clinical diagnosis of NE is significantly different from that of neurologically normal neonates; (3) to identify the source of NOS1 in umbilical cord blood; and (4) to simulate hypoxic events that may lead to NE and to evaluate production of NOS1 ex vivo.

\section{MATERIALS AND METHODS}

\section{Study Design}

We undertook a nested case-control study within a prospective cohort of neonates, $>35$ weeks of gestation; cohort has been described previously (12). Cases were neonates with NE and controls were age-matched newborns, admitted to the Johns Hopkins University Hospital (JHH) Neonatal Intensive Care Unit (NICU) between April 1, 2009 and May 30, 2014 (12). The Institutional Review Board at Johns Hopkins University School of Medicine approved this study, and all participants were anonymized.
Cases (Table 1) were neonates clinically diagnosed with moderate to severe NE, as defined by The American Academy of Pediatrics (5), who underwent whole-body cooling as per the JHH NICU institutional protocol which follows the recommendations of Shankaran et al. (22). Controls (Table 1), matched with the cases by gestational age (within 1 week), were neurologically normal neonates.

For the secondary analysis, we created a subset that met stringent criteria for NE cases and controls, based on clinical exam plus two additional criteria: umbilical cord arterial $\mathrm{pH}<7.0$ and base deficit $>12$ (Table 2$)(23,24)$. Neurologically normal neonates had a normal exam, umbilical artery $\mathrm{pH}>7.2$, and base deficit $<8$.

\section{Maternal and Neonatal Characteristics}

Maternal and neonatal records were reviewed, and pertinent clinical information was extracted. Data collected included infant sex, mode of delivery, gestational age, maternal age, parity, race, and three conditions suggested being risk factors for $\operatorname{NE}(25,26)$ : diagnosis of preeclampsia, clinical chorioamnionitis, and IUGR. Preeclampsia was defined as proteinuria and new onset hypertension. Clinical chorioamnionitis was defined as the presence of maternal fever with at least one other finding of fetal tachycardia,

TABLE 1 | Maternal demographics and perinatal outcomes for neonatal encephalopathy (NE) cases and normal controls.

\begin{tabular}{|c|c|c|c|}
\hline Characteristic & $\begin{array}{l}\text { Neurologically } \\
\text { normal controls } \\
(n=37)\end{array}$ & $\begin{array}{l}\text { NE cases } \\
(n=27)\end{array}$ & $P$ value \\
\hline $\begin{array}{l}\text { Gestational age at delivery } \\
\text { (weeks) }\end{array}$ & $39.6 \pm 1.0$ & $39.2 \pm 1.4$ & 0.70 \\
\hline Maternal age (years) & $26.5 \pm 5.7$ & $27 \pm 7.6$ & 0.63 \\
\hline Nulliparous & $17(61 \%)$ & $15(56 \%)$ & 0.70 \\
\hline White race & $7(28 \%)$ & $10(37 \%)$ & 0.33 \\
\hline Preeclampsia ${ }^{a}$ & $3(11 \%)$ & $2(7 \%)$ & 0.18 \\
\hline Clinical chorioamnionitis ${ }^{a}$ & $4(14 \%)$ & $3(11 \%)$ & 0.72 \\
\hline Intrauterine growth restriction ${ }^{a}$ & $3(11 \%)$ & $2(7 \%)$ & 0.97 \\
\hline Cesarean delivery & 15 (54\%) & $20(74 \%)$ & 0.11 \\
\hline Infant male sex & $18(64 \%)$ & 17 (63\%) & 0.92 \\
\hline
\end{tabular}

Data are presented as means and SD for the two continuous variables, and counts and percentages for the seven categorical variables.

${ }^{a}$ Risk factors associated with NE.

TABLE 2 | Stringent criteria for neonatal encephalopathy and neurologically normal neonates.

\begin{tabular}{ll}
\hline Encephalopathic neonates & Neurologically normal neonates \\
\hline At least one clinical criteria & Neurologically normal exam \\
- Lethargy & \\
- Stupor or coma & \\
- Decreased or no activity & \\
- Distal flexion or complete extension & \\
- Decerebrate posture & \\
- Hypotonia or flaccidity & \\
- Abnormal primitive reflexes & \\
- Abnormal autonomic nervous system & Umbilical cord pH $>7.2$ \\
- Seizures & Base deficit $<8$ \\
Umbilical cord pH $<7.0$ & \\
Base deficit $>12$ &
\end{tabular}


uterine tenderness, or purulent vaginal discharge. IUGR was defined as an estimated fetal weight less than the 10th percentile for gestational age with ultrasound performed within 3 weeks after delivery date.

\section{Collection of Umbilical Cord Blood for Determination of NOS1}

Venous umbilical cord plasma was collected from routinely discarded collected blood. Samples were stored at $-80^{\circ} \mathrm{C}$ until utilized.

\section{Ex Vivo Hypoxia of Umbilical Cord}

Umbilical cords were obtained from healthy full-term births within $4 \mathrm{~h}$. Methods previously described by Thomas et al. (27); briefly, in a sterile cell culture hood, umbilical cord was cut into 1-mm length thick slices. For normoxia, specimens were cultured in a conventional incubator preset at $37^{\circ} \mathrm{C}$ in a humidified atmosphere, containing $5 \% \mathrm{CO}_{2}$. Hypoxia was induced by a modular incubator chamber. To create gas hypoxia, specimens were first sealed in a humidified hypoxia incubator chamber system (Stemcell Technologies, Vancouver, BC, Canada) supplied with $1 \% \mathrm{O}_{2}$ at $37^{\circ} \mathrm{C}$ according to the manufacturer's instructions. After $3 \mathrm{~h}$ of incubation (simulating fetal hypoxic-ischemic event), umbilical cords were fixed with $4 \%$ paraformaldehyde (Affymetrix, Cleveland, OH, USA) overnight at $4^{\circ} \mathrm{C}$ for immunohistochemistry (IHC), or the tissue was trimmed close to the vein and artery followed by immediate storage at $-80^{\circ} \mathrm{C}$ for western blot. Culture media was also collected and frozen at $-80^{\circ} \mathrm{C}$.

\section{ELISA for NOS1}

ELISA of total NOS1 was performed according to the protocol established in our laboratory for indirect "sandwich" ELISA, optimized for highest sensitivity of human NOS1 in serum, amniotic fluid, or cell lysates. Briefly, an immobilized capture antibody for human NOS1 was coated overnight in 96-well microplate (R\&D, Minneapolis, MN, USA). After blocking and sample incubation, the unbound material was washed away, and a biotinylated detection antibody specific for human NOS1 ( $2 \mathrm{ng} / \mathrm{ml}$, Cell Signaling Tech, Beverley, MA, USA) was used to detect the target protein utilizing a standard streptavidin-HRP format using 3,3',5,5' -tetramethylbenzidine (TMB) as a substrate and subsequent reading at $450 \mathrm{~nm}$. Biotinylation as well as prior purification of detection antibodies were done using biotinylation and protein A/G purification kits according to manufacturer's recommendations (Pierce Biotechnology, Rockford, IL, USA). Recombinant purified NOS1 was purchased from Enzo Life Sciences, Inc. (Farmingdale, NY, USA) for standard curve. Average for blank readings was subtracted from the averages for the duplicate readings for each sample, and total NOS1 concentrations of each sample were determined using log-linear regression. ELISA was further validated with $\mathrm{JHH}$ population with a separate group of samples.

\section{Immunohistochemistry}

Using a cryostat (Leica, Buffalo Grove, IL, USA), umbilical cords were sectioned into $20-\mu \mathrm{m}$ thick slices. Sections were incubated overnight at $4^{\circ} \mathrm{C}$ with primary antibodies in PBS containing 0.5\% Triton X-100 (Sigma, St. Louis, MO, USA) after blocking with $10 \%$ goat serum. The following primary antibodies were used: rabbit against NOS1 (1:1,000, Millipore, Billerica, MA, USA), NOS2 (1:20, Novus, Littleton, CO, USA), NOS3 (1:100, Abcam, Cambridge, MA, USA), and mouse against CD34 (1:500, Millipore, Billerica, MA, USA). CD34 was used to characterize endothelial cells in human UC. The next day, sections were rinsed with PBS, and then incubated with fluorescent secondary antibodies diluted in 1:500 for $3 \mathrm{~h}$ at room temperature. The following antibodies were used for immunofluorescence: goat antirabbit DyLight 488 (Abcam, Cambridge, MA, USA) and donkey anti-mouse Alexa Fluor 568 (Life Technologies, Grand Island, NY, USA). The sections were further stained with DAPI (Roche, Indianapolis, IN, USA) for $2 \mathrm{~min}$ at room temperature followed by mounting with Fluromount-G (eBioscience, San Diego, CA, USA). Images were attained using Axioplan 2 Imaging system (Carl Zeiss, Thornwood, NY, USA). To exclude a possibility that NOS1 positive cells were blood cells or of stem cell origin, double staining of NOS1/CD44 (Abcam, Cambridge, MA, USA) and NOS1/CD45 (Abcam, Cambridge, MA, USA) was also conducted.

To further confirm the distribution of NOS1 in human UC, another four NOS1 from hosts of rabbit or mouse (Cell Signaling, Beverly, MA, USA; Life Technologies, Frederick, MD, USA; Abcam, Cambridge, MA, USA; and BD Biosciences, Franklin Lakes, New Jersey, USA), one NOS2 (Abcam, Cambridge, MA, USA), and one NOS3 (Abcam, Cambridge, MA, USA) antibodies were utilized to exam the expression together with CD34 antibody. The secondary antibodies were used the same as described above.

\section{SDS-PAGE and Western Blotting}

The following were used as primary antibodies: rabbit against NOS1 (Millipore, Billerica, MA, USA), NOS2 (Novus, Littleton, CO, USA), NOS3 (Abcam, Cambridge, MA, USA) and rabbit anti $\beta$-actin (Abcam, Cambridge, MA, USA). Extracted protein $(50 \mu \mathrm{g})$ was separated by SDS-PAGE using $4-15 \%$ gels (Bio-Rad Laboratories, Hercules, CA, USA) and then electro-transferred onto nitrocellulose membranes (Bio-Rad Laboratories, Hercules, CA, USA). Membranes were blocked with 10\% BSA (SigmaAldrich, St. Louis, MO, USA) in Tris-buffered saline $+0.1 \%$ Tween-20 (TBS-T, pH7.5), incubated with primary antibodies in TBS-T containing $10 \%$ BSA for $1 \mathrm{~h}$ at room temperature, and washed in TBS-T. Goat anti-rabbit IR Dye-800CW (Li-Cor, Lincoln, NB, USA) was used as the secondary antibody. Imaging was performed using the Li-Cor Odyssey Near Infra Red System and analyzed using Image Studio Software (Li-Cor).

\section{Quantitative PCR (qPCR)}

Vein and artery tissue were dissected from umbilical cords and frozen at $-80^{\circ} \mathrm{C}$ until used. The tissue samples were homogenized in RLT buffer (Qiagen, Valencia, CA, USA) for $60 \mathrm{~s}$ using a BeadBug microtube homogenizer (Benchmark Scientific, Edison, NJ, USA) with $0.5-\mathrm{mm}$ beads. Cord blood was collected into acid citrate dextrose tubes (BD Biosciences, Franklin Lakes, NJ, USA) and centrifuged at $400 \times g$ for $10 \mathrm{~min}$ at room temperature. RNA was prepared from the umbilical cord homogenates and 
cord blood leukocytes with RNEasy Mini Kit (Qiagen, Valencia, CA, USA), and cDNA was prepared using cDNA Synthesis Kit (Bio Rad, Hercules, CA, USA). qPCR was performed in triplicate in 20- $\mu$ l reactions for 40 cycles, using the manufacturer's suggested protocols for temperature cycling (Bio Rad, Hercules, CA, USA). The reactions were run on a CFX384 Touch Real-Time PCR Detection System (Bio Rad, Hercules, CA, USA), using SensiFAST Probe No-ROX (Bioline, Taunton, MA, USA). Primers used were obtained from Integrated DNA Technologies (Coralville, IA, USA) for NOS1 (Fwd: AGACGCACGAAGATAGTTGAC, Rev: CCGAAGCTCCAGAACTCAC, Probe:/56-AM/TCCTTAGCC/ ZEN/GTCAAAACCTCCAGAG/3IABkFQ), NOS2 (Fwd: ACTTCCACTTGCTGTACTCTG, Rev: CACCTACTTCCTG GACATCAC, Probe: CTGCTGCTCCAAAAGCTGGCC), and NOS3 (Fwd: ACGATGGTGACTTTGGCTA, Rev: TGGAGGATG TGGCTGTCT, Probe: CAGTGGAAATCAACGTGGCCGTG) or SsoAdvanced Universal SYBR Green Supermix (Bio-Rad) for Eukaryotic 18S Ribosomal RNA (Life Technologies, Grand Island, NY, USA). Data analysis was performed with CFX Manager Software (Bio Rad).

\section{Statistical Analysis}

Data were compared using Student's $t$-test, Mann-Whitney $U$ test, or Fisher exact test, when appropriate. Two-tailed Student's $t$-test or Mann-Whitney $U$ test was used subsequently to determine whether data were normally distributed. Fold change of NOS1 level over mean control was calculated.

To determine the ability of NOS1 to predict NE, and the optimal cut points for NOS1, we created receiver operator characteristic (ROC) curves. Sensitivity and specificity were determined for each of these points. Statistical analyses were performed using Stata10 (StataCorp LP, College Station, TX, USA).

\section{RESULTS}

\section{Study Population}

Umbilical cord blood samples of $27 \mathrm{NE}$ cases and 37 controls were used. There were no significant differences between cases and controls in: (i) maternal demographic parameters (gestational age at delivery, maternal age, nulliparous, and white race), (ii) incidence of adverse perinatal outcomes (preeclampsia, clinical chorioamnionitis, intrauterine growth restriction), or (iii) cesarean delivery or infant sex (Table 1). For an exploratory binding assay to detect NOS1 in umbilical cord blood of neonates, $15 \mathrm{NE}$ and 24 control neonates were utilized. Eight neonates met stringent criteria for NE and were matched to ten control neonates (Table 2). Another $12 \mathrm{NE}$ and 13 controls were utilized to validate the exploratory results by ELISA. We demonstrated the presence of NOS1 in the umbilical cord blood of all 64 neonates.

\section{Level of NOS1 in Neonates with NE and in Neurologically Normal Neonates}

In our investigational study, as determined by relative absorbance values of ELISA (Figure 1A), the level of NOS1 in umbilical cord blood was significantly higher in NE cases (mean $=1.392 \mathrm{AU}$, 95\% CI 1.04-1.74) than in the controls (mean $=1.00 \mathrm{AU}, 95 \%$ CI 0.77-1.23). Since these values were generated from a noncommercial ELISA (prepared in our laboratory and originally without standards/standard curve), these findings were validated by a separate experiment to determine NOS1 concentration, involving separate set of samples and standards/standard curve $(\mathrm{NE}:$ mean $=1.32 \mathrm{ng} / \mathrm{ml}$; Control: mean $=0.03 \mathrm{ng} / \mathrm{ml} ; p<0.05$, Student's $t$-test; Figure 1B).

\section{Stringent Criteria Subset}

In umbilical cord blood, the mean relative absorbance of NOS1 level was significantly higher (Figure 2A, $p<0.05$, Student's $t$-test) in the neurologically at-risk neonates meeting stringent criteria (mean $=2.006 \mathrm{AU}, 95 \% \mathrm{CI} 1.425-2.587$ ) than in the neurologically normal control neonates (mean $=1.000 \mathrm{AU}, 95 \%$ CI 0.493-1.507). Within the group of neonates meeting stringent criteria, NOS1 was predictive of NE. The ROC curve yielded an AUC of 0.84 for NOS1 prediction of NE (Figure 2B).

\section{NOS1 Expression in Endothelial Cells of Human Umbilical Vein}

To localize NOS1 in the human umbilical cord, IHC for NOS1 and CD34 were performed. IHC for CD34 revealed a distribution of endothelial cells in the umbilical cord (28). NOS1 was
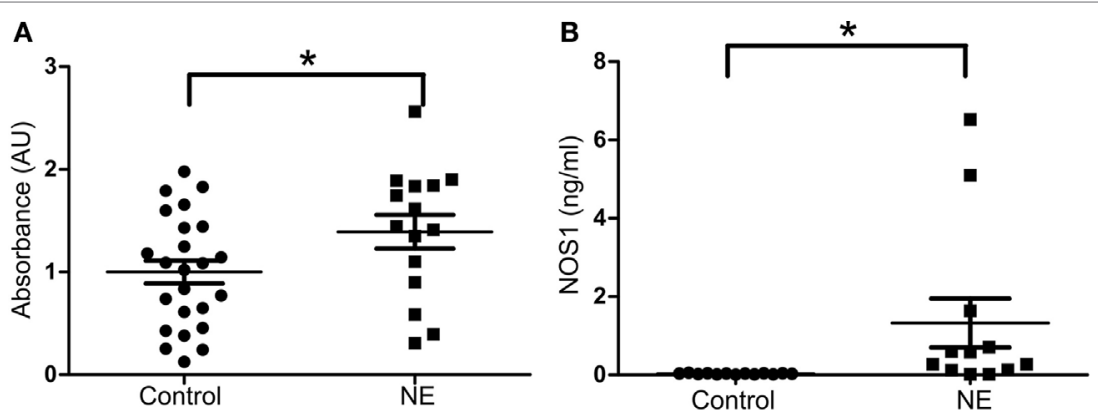

FIGURE 1 | Expression of NOS1 in umbilical cord blood. (A) Bar plot of relative absorbance in NOS1 level compared to controls based on exploratory NOS1-binding assay ( $n=15$ NE cases and 24 controls, Student's $t$-test; ${ }^{*} p<0.05$ ). (B) NOS1 concentrations were determined by ELISA using a separate set of patients ( $n=12$ NE cases and 13 controls; Student's $t$-test; $\left.{ }^{*} p<0.05\right)$. Data are reported as means \pm SEM. 

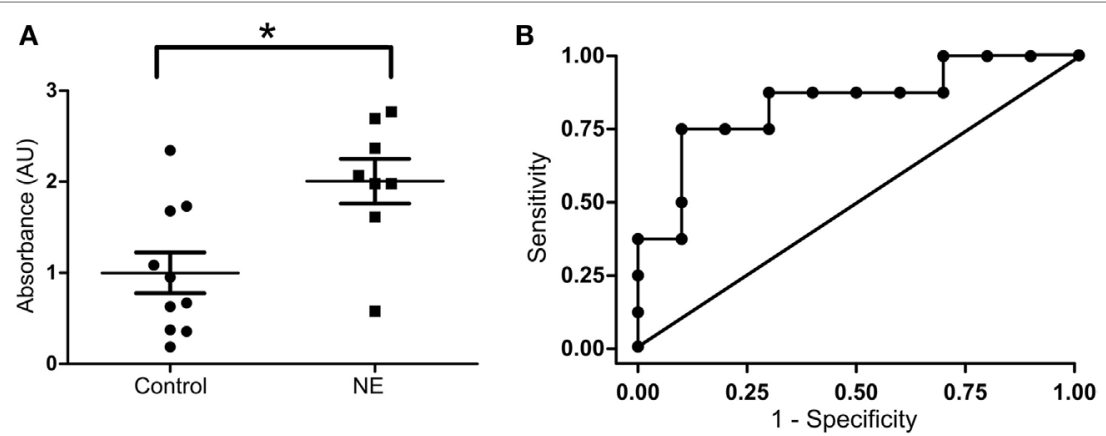

FIGURE 2 | NOS1 expression in NE patients classified by stringent criteria. (A) Relative absorbance of NOS1 expression compared to controls based on exploratory NOS1 binding assay ( $n=8 \mathrm{NE}$ cases and 10 controls, Student's $t$-test; $\left.{ }^{*} p<0.05\right)$. Bars represent mean \pm SEM. (B) Receiver operator characteristic (ROC) curve for NOS1 expression. Area under the curve equals 0.84 .

expressed mainly in the form of fine granules in the cytoplasm and, occasionally, in cell membrane. There was a co-localization of NOS1 and CD34 in the umbilical vein, as demonstrated by double-positive staining (Figure 3A, top panel). Neither CD44 nor CD45 cells was co-localized with NOS1 (data not shown). There was no clear NOS1 expression in umbilical artery (Figure 3A, bottom panel). Western blot further confirmed IHC results with significant expression of NOS1 in umbilical vein (Figures 3B,C, Student's $t$-test, $p<0.05)$.

Confirmatory analysis examining expression of NOS1, NOS2 (inducible NOS), and NOS3 (endothelial NOS) at mRNA and protein level in umbilical cord and leukocytes were also performed. NOS1 was only detected in umbilical cord vein (Table 3), while NOS2 and NOS3 expression was detected by IHC and qPCR in both umbilical cord vein and artery. Transcripts for NOS1, NOS2, and NOS3 were not detected in cord blood leukocytes (Table 3) using qPCR.

To further ascertain a differential expression as well as to validate the specificity of the antibodies used, another four NOS1, one NOS2, and one NOS3 antibodies were used in IHC. Similar to the above results, NOS1 expression was detected in umbilical cord vein but not in artery (Figure S1A in Supplementary Material). NOS2 (Figure S1B in Supplementary Material) and NOS3 (Figure S1C in Supplementary Material) were detected in both umbilical cord vein and artery.

\section{Western Blot and ELISA Analyses of NOS1 Expression in Ex Vivo Umbilical Cord Culture}

To investigate whether hypoxia induces a NOS1 response in venous endothelial cells, umbilical cord slices were cultured in a hypoxia chamber under $1 \% \mathrm{O}_{2}$. After $3 \mathrm{~h}$ of culture (simulating a clinical hypoxic event), the co-localization of CD34 and NOS1 increased in the hypoxic group compared with normoxic control, by IHC (Figure 4A) and by western blot (Figures 4B,C, $p<0.05$, Student's $t$-test). We further measured NOS1 production in culture supernatant and, after $3 \mathrm{~h}$ of hypoxia, NOS1 increased significantly following hypoxic conditions (Figure 4D, Student's t-test, $p<0.01)$.

\section{DISCUSSION}

Our study is the first to demonstrate that NOS1, a marker of oxidative stress, is a potential early bedside clinical biomarker of NE in umbilical cord blood. In our study, the levels of NOS1 were significantly higher in the umbilical cord blood of neonates with clinical encephalopathy than in normal controls. Furthermore, our ex vivo experiments confirmed that NOS1 was increased with hypoxia in venous endothelial cells of umbilical cord.

There are a few biomarkers relative to neonatal brain injury. NOS1 is currently the only early biomarker that is available to aid in diagnosis of NE at delivery based on umbilical cord blood. Massaro et al. evaluated the serum biomarkers S100B and NSE in neonates treated with hypothermia and found them to be associated with clinical encephalopathy and MRI change (3), consistent with prior studies $(13-15,29)$. None of the biomarkers were used for diagnosis or triage of neonates to therapy. In addition, the first time point evaluated was at initiation of cooling; information was not available prior to initiation of cooling and could not assist with triage to therapy which represents a limitation of their study. Furthermore, others evaluated adrenomedullin and activin A have been evaluated as biomarkers of NE in neonatal serum but similarly have not been tested in umbilical cord blood (30). In Ennen's studies (12), GFAP in serum at 24 and $48 \mathrm{~h}$ after birth was predictive of brain injury on MRI in encephalopathic neonates. Similarly to other markers discussed, at birth, umbilical cord blood GFAP levels were not predictive of brain injury.

We used ELISA for NOS1, which may present as a new, fast, and easy method to perform clinical test due to high levels (nanograms) of NOS1 presented in umbilical cord blood. As the AUC of NOS1 test is perhaps superior as compared to current clinical criteria for diagnosis of NE [AUC 0.81 (31)], we speculate that implementation of this test will improve sensitivity and specificity of timely clinical diagnosis as it can be used concomitantly. Results of this test can be provided within the first several hours of life, allowing for early diagnosis and triage to therapy such as hypothermia and other novel therapeutics. One novel use for such clinical test would include early identification of which neonates 

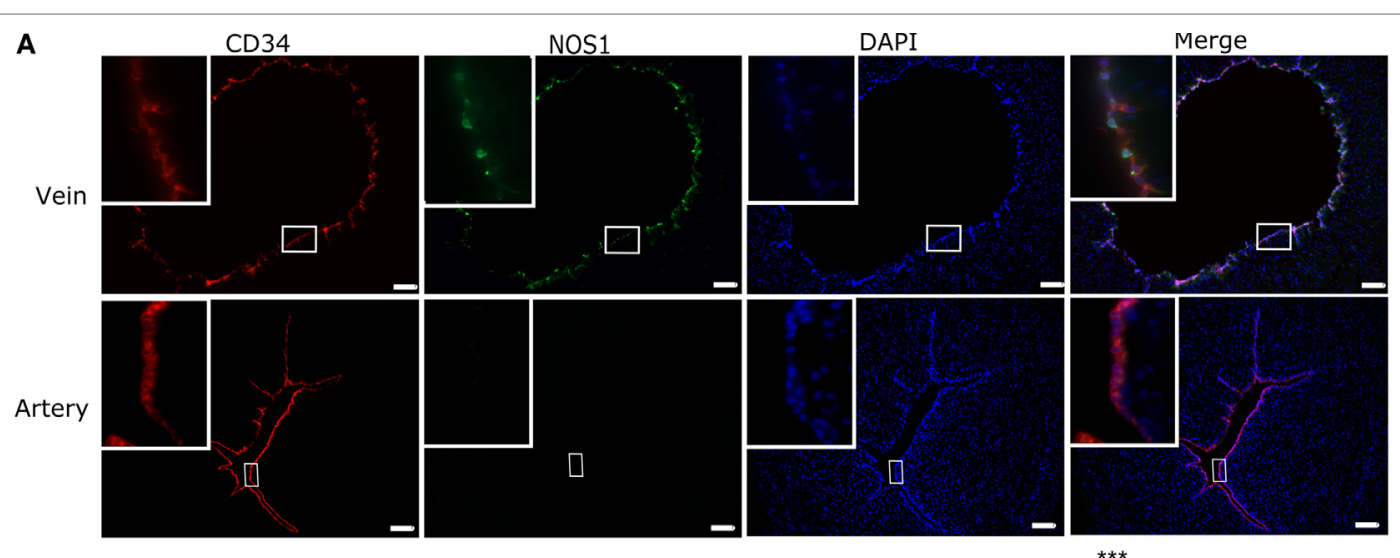

B

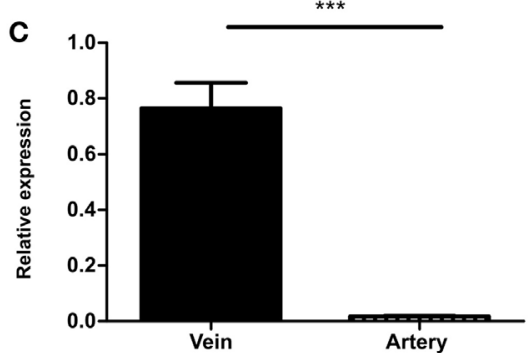

FIGURE 3 | NOS1 Expression in vessels of human umbilical cord. (A) Immunostaining of CD34 and NOS1 in endothelial cells of vein and artery of umbilical cord ( $n=4$ per group). (B) Protein expression of NOS1 in vein and artery of umbilical cord by western blot. (C) Densitometry analysis of western blot imaging ( $n=5$ per group; Student's $t$-test; $\left.{ }^{*} p<0.05\right)$. Scale bars $=100 \mu \mathrm{m}$.

TABLE 3 | Detection of NOS1, NOS2, and NOS3 in umbilical cord vein and artery.

\begin{tabular}{|c|c|c|c|c|c|c|}
\hline & \multicolumn{3}{|c|}{$\begin{array}{l}\text { Immunohistochemistry } \\
\text { (IHC) }\end{array}$} & \multicolumn{3}{|c|}{ Quantitative PCR } \\
\hline & NOS1 & NOS2 & NOS3 & NOS1 & NOS2 & NOS3 \\
\hline Umbilical cord artery & $-(0 / 4)$ & $+(4 / 4)$ & $+(4 / 4)$ & $-(0 / 4)$ & $+(4 / 4)$ & $+(3 / 4)$ \\
\hline Umbilical cord vein & $+(4 / 4)$ & $+(4 / 4)$ & $+(4 / 4)$ & $+(3 / 4)$ & $+(4 / 4)$ & $+(3 / 4)$ \\
\hline $\begin{array}{l}\text { Cord blood } \\
\text { leukocytes }\end{array}$ & & & & $-(0 / 4)$ & $-(0 / 4)$ & $-(0 / 4)$ \\
\hline
\end{tabular}

-, negative expression; +, positive expression. There were four samples for each group. In brackets, numerators are the numbers tested negative or positive expression by $I \mathrm{HC}$ or quantitative PCR.

that might benefit from storage of umbilical cord blood for later stem cell transplantation and would result in timely collection of umbilical cord blood.

Neonatal encephalopathy is a rare diagnosis and MRI diagnosis of actual brain injury in this group is an infrequent finding. The consequences of NE including death and neurodevelopmental disability can be devastating for the child and family. While one critique of our study could be a small sample size, the nested case-control study represents a valuable sample, given that $\mathrm{NE}$ is rare, even in our referral university center. However, our data are confirmed with simulation of hypoxia ex vivo, and this is an advantage of our study. Further research will concentrate on collecting information about childhood outcomes of these at-risk neonates with neurodevelopmental testing at $1-3$ years of age.

Our study has several strengths. An ELISA assay quantified the level of NOS1, and this test can be used in a clinical setting to inform clinical decisions early in neonatal life. The results are reproducible, and more stringent criteria for encephalopathy yields improved prediction of NOS1 for NE. In addition to identifying neonates at risk of brain injury, a biomarker would timely predict which infants are at risk for brain injury and which are most likely to have long-term neurologic sequelae. Moreover, our study identifies a source of NOS1, and, using experimental conditions that mimic short-term hypoxic environments, we are able to observe similar findings ex vivo.

Perinatal hypoxia-ischemia is an important cause of NE (50$80 \%$ ) (32). Nitric oxide signaling has been demonstrated to have an important role in hypoxia-induced pathological responses as all NOS isoforms critically require oxygen for activity (33). Physiologically, NOS1 depends more on oxygen and has higher rates of nitric oxide biosynthesis (34) than NOS2 and NOS3. Both NOS1 mRNA and protein expression are regulated by $\mathrm{O}_{2}$ at transcriptional and translational level $(35,36)$, and therefore, it may be more sensitive to hypoxia. Corollary to that, in our study, NOS1 significantly increased in umbilical cord blood of moderate and severe NE neonates compared to control.

To investigate a plausible origin of NOS1 in umbilical cord blood, we performed a survey of all possible sources of NOS1 in umbilical cord blood and umbilical cord. We found that NOS1 was present in endothelial cells of human umbilical cord 


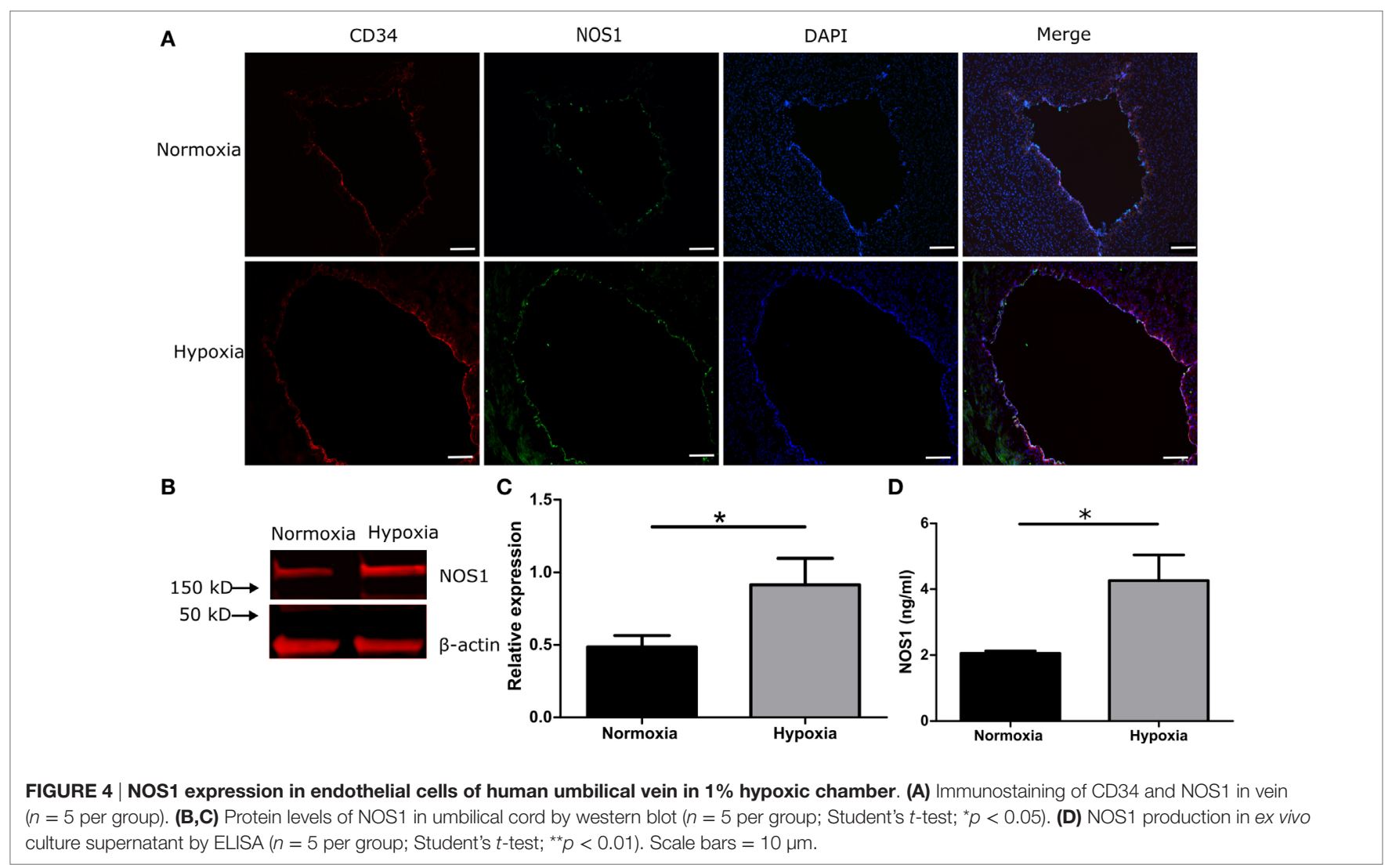

vein but not in umbilical cord artery. While our results are in keeping with a previous study (20) that showed NOS1 expression in vein of human umbilical cord by IHC, our study is the first to demonstrate a differential expression of this enzyme in umbilical vein and artery. In the adult vascular system, NOS1and NOS3-derived nitric oxide is thought to play a major role in changes of vascular arterial tone $(37,38)$. In addition to regulating vascular tone, nitric oxide is an important signaling molecule in the nervous system and the immune system $(39,40)$. We speculate that endothelial NOS1 expression in umbilical vein may be a conserved mechanism that contributes to the fetal response to hypoxia and inflammation. Further studies to clarify the role of umbilical cord NOS1 during hypoxic and inflammatory conditions are needed.

In conclusion, we speculate that NOS1 is a novel, early biomarker for early identification of $\mathrm{NE}$ that can be used in determining NE together with clinical manifestations to improve diagnosis, allowing for better prognostic information and earlier interventions.

\section{ETHICS STATEMENT}

This study was carried out in accordance with the recommendations of Johns Hopkins University Hospital (JHH) Neonatal Intensive Care Unit and the protocol was approved by The Institutional Review Board at Johns Hopkins University School of Medicine. All participants were anonymized.

\section{AUTHOR CONTRIBUTIONS}

JL and JR performed the experiments, analyzed data, and wrote the manuscript. SY analyzed data and wrote the manuscript. RB and SM perfumed the experiments and analyzed data. CP, EN, $\mathrm{AZ}, \mathrm{FN}, \mathrm{DO}, \mathrm{EG}$, and MJ designed the experiments and wrote the manuscript. IB designed and performed the experiments, analyzed data, and wrote the manuscript.

\section{FUNDING}

This work was supported by Sheikh Abdullah Bugshan fund 80034548 (IB), NICHD K08HD073315 (IB), NINDS NS28208 (MJ), and NICHD R01HD086058 (FN).

\section{SUPPLEMENTARY MATERIAL}

The Supplementary Material for this article can be found online at http://journal.frontiersin.org/article/10.3389/fped.2017.00112/ full\#supplementary-material.

FIGURE S1 | Distribution of NOS1, NOS2, and NOS3 expression in umbilical cord. (A) Representative image of NOS1 (green) in umbilical cord using four different antibodies. NOS1 was expressed in the venous endothelial cells (red) of human umbilical cord, but not in the artery. (B) NOS2 was expressed in both endothelial cells (red) of vein and artery. (C) NOS3 (green) was also expressed in both endothelial cells (red) of vein and artery. Scale bars $=10 \mu \mathrm{m}$. 


\section{REFERENCES}

1. Pierrat V, Haouari N, Liska A, Thomas D, Subtil D, Truffert P, et al. Prevalence, causes, and outcome at 2 years of age of newborn encephalopathy: population based study. Arch Dis Child Fetal Neonatal Ed (2005) 90:F257-61. doi:10.1136/ adc.2003.047985

2. Graham EM, Ruis KA, Hartman AL, Northington FJ, Fox HE. A systematic review of the role of intrapartum hypoxia-ischemia in the causation of neonatal encephalopathy. Am J Obstet Gynecol (2008) 199:587-95. doi:10.1016/j. ajog.2008.06.094

3. Massaro AN, Jeromin A, Kadom N, Vezina G, Hayes RL, Wang KK, et al. Serum biomarkers of MRI brain injury in neonatal hypoxic ischemic encephalopathy treated with whole-body hypothermia: a pilot study. Pediatr Crit Care Med (2013) 14:310-7. doi:10.1097/PCC.0b013e3182720642

4. Lee AC, Kozuki N, Blencowe H, Vos T, Bahalim A, Darmstadt GL, et al. Intrapartum-related neonatal encephalopathy incidence and impairment at regional and global levels for 2010 with trends from 1990. Pediatr Res (2013) 74(Suppl 1):50-72. doi:10.1038/pr.2013.206

5. Executive summary: neonatal encephalopathy and neurologic outcome, second edition. Report of the American College of Obstetricians and Gynecologists' Task Force on Neonatal Encephalopathy. Obstet Gynecol (2014) 123:896-901. doi:10.1097/01.AOG.0000445580.65983.d2

6. Epelman M, Daneman A, Kellenberger CJ, Aziz A, Konen O, Moineddin R, et al. Neonatal encephalopathy: a prospective comparison of head US and MRI. Pediatr Radiol (2010) 40:1640-50. doi:10.1007/s00247-010-1634-6

7. Miller SP, Ramaswamy V, Michelson D, Barkovich AJ, Holshouser B, Wycliffe N, et al. Patterns of brain injury in term neonatal encephalopathy. J Pediatr (2005) 146:453-60. doi:10.1016/j.jpeds.2004.12.026

8. Committee on Fetus and Newborn, Papile LA, Baley JE, Benitz W, Cummings J, Carlo WA, et al. Hypothermia and neonatal encephalopathy. Pediatrics (2014) 133(6):1146-50. doi:10.1542/peds.2014-0899

9. Azzopardi DV, Strohm B, Edwards AD, Dyet L, Halliday HL, Juszczak E, et al. Moderate hypothermia to treat perinatal asphyxial encephalopathy. $N$ Engl J Med (2009) 361:1349-58. doi:10.1056/NEJMoa0900854

10. Xiong T, Qu Y, Mu D, Ferriero D. Erythropoietin for neonatal brain injury: opportunity and challenge. Int J Dev Neurosci (2011) 29:583-91. doi:10.1016/j. ijdevneu.2010.12.007

11. Liao Y, Cotten M, Tan S, Kurtzberg J, Cairo MS. Rescuing the neonatal brain from hypoxic injury with autologous cord blood. Bone Marrow Transplant (2013) 48:890-900. doi:10.1038/bmt.2012.169

12. Ennen CS, Huisman TA, Savage WJ, Northington FJ, Jennings JM, Everett AD, et al. Glial fibrillary acidic protein as a biomarker for neonatal hypoxicischemic encephalopathy treated with whole-body cooling. Am JObstet Gynecol (2011) 205(251):e251-7. doi:10.1016/j.ajog.2011.06.025

13. Nagdyman N, Grimmer I, Scholz T, Muller C, Obladen M. Predictive value of brain-specific proteins in serum for neurodevelopmental outcome after birth asphyxia. Pediatr Res (2003) 54:270-5. doi:10.1203/01.PDR. 0000072518.98189.A0

14. Thorngren-Jerneck K, Alling C, Herbst A, Amer-Wahlin I, Marsal K. S100 protein in serum as a prognostic marker for cerebral injury in term newborn infants with hypoxic ischemic encephalopathy. Pediatr Res (2004) 55:406-12. doi:10.1203/01.PDR.0000106806.75086.D3

15. Celtik C, Acunas B, Oner N, Pala O. Neuron-specific enolase as a marker of the severity and outcome of hypoxic ischemic encephalopathy. Brain Dev (2004) 26:398-402. doi:10.1016/j.braindev.2003.12.007

16. Rao S, Lin Z, Drobyshevsky A, Chen L, Ji X, Ji H, et al. Involvement of neuronal nitric oxide synthase in ongoing fetal brain injury following near-term rabbit hypoxia-ischemia. Dev Neurosci (2011) 33:288-98. doi:10.1159/000327241

17. Muramatsu K, Sheldon RA, Black SM, Tauber M, Ferriero DM. Nitric oxide synthase activity and inhibition after neonatal hypoxia ischemia in the mouse brain. Brain Res Dev Brain Res (2000) 123:119-27. doi:10.1016/S0165-3806(00)00088-2

18. Thomas GD, Sander M, Lau KS, Huang PL, Stull JT, Victor RG. Impaired metabolic modulation of alpha-adrenergic vasoconstriction in dystrophin-deficient skeletal muscle. Proc Natl Acad Sci U S A (1998) 95:15090-5. doi:10.1073/pnas.95.25.15090

19. Kobzik L, Reid MB, Bredt DS, Stamler JS. Nitric oxide in skeletal muscle. Nature (1994) 372:546-8. doi:10.1038/372546a0
20. Bachetti T, Comini L, Curello S, Bastianon D, Palmieri M, Bresciani G, et al. Co-expression and modulation of neuronal and endothelial nitric oxide synthase in human endothelial cells. J Mol Cell Cardiol (2004) 37:939-45. doi:10.1016/j.yjmcc.2004.07.006

21. Carnicer R, Crabtree MJ, Sivakumaran V, Casadei B, Kass DA. Nitric oxide synthases in heart failure. Antioxid Redox Signal (2013) 18:1078-99. doi: 10.1089 /ars.2012.4824

22. Shankaran S, Laptook AR, Ehrenkranz RA, Tyson JE, McDonald SA, Donovan EF, et al. Whole-body hypothermia for neonates with hypoxic-ischemic encephalopathy. N Engl J Med (2005) 353:1574-84. doi:10.1056/ NEJMcps050929

23. Yeh P, Emary K, Impey L. The relationship between umbilical cord arterial $\mathrm{pH}$ and serious adverse neonatal outcome: analysis of 51,519 consecutive validated samples. BJOG (2012) 119:824-31. doi:10.1111/j.1471-0528.2012.03335.x

24. American Collge of Obstetricians and Gynecologists (ACOG). Neonatal encephalopathy and cerebral palsy: executive summary. Obstet Gynecol (2004) 103:780-1. doi:10.1097/01.AOG.0000120142.83093.30

25. Nelson KB, Bingham P, Edwards EM, Horbar JD, Kenny MJ, Inder T, et al. Antecedents of neonatal encephalopathy in the Vermont Oxford Network Encephalopathy Registry. Pediatrics (2012) 130:878-86. doi:10.1542/ peds.2012-0714

26. Badawi N, Kurinczuk JJ, Keogh JM, Alessandri LM, O’Sullivan F, Burton PR, et al. Antepartum risk factors for newborn encephalopathy: the Western Australian case-control study. BMJ (1998) 317:1549-53. doi:10.1136/ bmj.317.7172.1549

27. Iftimia-Mander A, Hourd P, Dainty R, Thomas RJ. Mesenchymal stem cell isolation from human umbilical cord tissue: understanding and minimizing variability in cell yield for process optimization. Biopreserv Biobank (2013) 11:291-8. doi:10.1089/bio.2013.0027

28. Fan CL, Li Y, Gao PJ, Liu JJ, Zhang XJ, Zhu DL. Differentiation of endothelial progenitor cells from human umbilical cord blood CD $34+$ cells in vitro. Acta Pharmacol Sin (2003) 24:212-8.

29. Nagdyman N, Komen W, Ko HK, Muller C, Obladen M. Early biochemical indicators of hypoxic-ischemic encephalopathy after birth asphyxia. Pediatr Res (2001) 49:502-6. doi:10.1203/00006450-200104000-00011

30. Gazzolo D, Abella R, Marinoni E, di Iorio R, Li Volti G, Galvano F, et al. New markers of neonatal neurology.J Matern Fetal Neonatal Med(2009)22(Suppl3): 57-61. doi:10.1080/14767050903181468

31. Thayyil S, Chandrasekaran M, Taylor A, Bainbridge A, Cady EB, Chong WK, et al. Cerebral magnetic resonance biomarkers in neonatal encephalopathy: a meta-analysis. Pediatrics (2010) 125:e382-95. doi:10.1542/peds. 2009-1046

32. Volpe JJ. Neonatal encephalopathy: an inadequate term for hypoxic-ischemic encephalopathy. Ann Neurol (2012) 72:156-66. doi:10.1002/ana.23647

33. Stuehr DJ. Mammalian nitric oxide synthases. Biochim Biophys Acta (1999) 1411:217-30. doi:10.1016/S0005-2728(99)00016-X

34. Stuehr DJ, Santolini J, Wang ZQ, Wei CC, Adak S. Update on mechanism and catalytic regulation in the NO synthases. J Biol Chem (2004) 279:36167-70. doi:10.1074/jbc.R400017200

35. Prabhakar NR, Pieramici SF, Premkumar DR, Kumar GK, Kalaria RN. Activation of nitric oxide synthase gene expression by hypoxia in central and peripheral neurons. Brain Res Mol Brain Res (1996) 43:341-6. doi:10.1016/ S0169-328X(96)00222-7

36. Huang J, Tamisier R, Ji E, Tong J, Weiss WJ. Chronic intermittent hypoxia modulates nNOS mRNA and protein expression in the rat hypothalamus. Respir Physiol Neurobiol (2007) 158:30-8. doi:10.1016/j.resp.2007.03.010

37. Li C, Ruan L, Sood SG, Papapetropoulos A, Fulton D, Venema RC. Role of eNOS phosphorylation at Ser-116 in regulation of eNOS activity in endothelial cells. Vascul Pharmacol (2007) 47:257-64. doi:10.1016/j.vph.2007. 07.001

38. Seddon MD, Chowienczyk PJ, Brett SE, Casadei B, Shah AM. Neuronal nitric oxide synthase regulates basal microvascular tone in humans in vivo. Circulation (2008) 117:1991-6. doi:10.1161/CIRCULATIONAHA. 107.744540

39. Nagy G, Koncz A, Perl A. T cell activation-induced mitochondrial hyperpolarization is mediated by $\mathrm{Ca}^{2+}$ - and redox-dependent production of nitric oxide. J Immunol (2003) 171:5188-97. doi:10.4049/jimmunol.171.10.5188 
40. Kano T, Shimizu-Sasamata M, Huang PL, Moskowitz MA, Lo EH. Effects of nitric oxide synthase gene knockout on neurotransmitter release in vivo. Neuroscience (1998) 86:695-9. doi:10.1016/S0306-4522(98)00179-1

Conflict of Interest Statement: The authors declare that the research was conducted in the absence of any commercial or financial relationships that could be construed as a potential conflict of interest.
Copyright (c) 2017 Lei, Paules, Nigrini, Rosenzweig, Bahabry, Farzin, Yang, Northington, Oros, McKenney, Johnston, Graham and Burd. This is an open-access article distributed under the terms of the Creative Commons Attribution License (CC BY). The use, distribution or reproduction in other forums is permitted, provided the original author(s) or licensor are credited and that the original publication in this journal is cited, in accordance with accepted academic practice. No use, distribution or reproduction is permitted which does not comply with these terms. 